\title{
PROSPECÇÃO TECNOLÓGICA NA CONCESSÃO DE PATENTES NO BRASIL: ESTUDO DE CASO EM MEDICAMENTOS IMUNOSSUPRESSORES DE TRANSPLANTE DE ÓRGÃOS
}

\author{
Autoria: \\ Jussanã Cristina de Abreu - Doutoranda, PPED/IE/UFRJ e Anvisa (Coordenação de propriedade \\ intelectual).E-mail:jussana@gmail.com. \\ Julia Paranhos - Professora Adjunta, IE/UFRJ. E-mail: juliaparanhos@ie.ufrj.br.
}

\section{RESUMO}

O trabalho objetiva aplicar uma metodologia que visa subsidiar a operacionalização da priorização da análise técnica de pedidos de patentes pelo Instituto Nacional de Propriedade Industrial (INPI) e Agência Nacional de Vigilância Sanitária (Anvisa) baseada no instrumental teórico-prático de prospecção tecnológica. A priorização do exame de patentes é uma das linhas de gestão do backlog de patentes normatizada no INPI, com potencial de otimizar a morosidade da concessão de patentes farmacêuticas no Brasil e seus efeitos deletérios no Sistema Nacional de Inovação (SNI) e no Sistema Único de Saúde (SUS), influindo na dinâmica de inovação do setor farmacêutico nacional, foco dos recentes planos de desenvolvimento nacional. Foi utilizada a técnica de mineração de dados em ciência e tecnologia (tech mining) num estudo de caso em tecnologias de interesse do Ministério da Saúde (MS), atuais e futuras, no campo tecnológico dos medicamentos imunossupressores de transplante de órgãos sólidos. Foram identificados 48 pedidos de patentes aptos ao exame prioritário no país e 21 produtos futuros no campo tecnológico supracitado. Constatou-se ainda a ausência de uma política explicita que delineie o papel ocupado pelo subsistema de concessão de patentes farmacêuticas, compromete as iniciativas políticas vigentes pelo que foram tecidas recomendações aos operadores das políticas públicas no país.

Palavras-chaves: exame de patentes, prospecção tecnológica, indústria farmacêutica, mineração de dados em ciência e tecnologia.

\begin{abstract}
The objective of this paper is to apply a methodology that aims to aid the operationalization of prioritization of patent application technical analysis by INPI (National Institute of Intellectual Property) and Anvisa (Brazilian Health Regulatory Agency), based in the theoretical-practical technological prospection tools. The prioritization of patents examination is one of the lines of patents backlog management ruled by the Institute with potential to optimize the slowdown of pharmaceutical patents concession and its deleterious effects in the National Innovation System (NIS) and Brazilian Health System (BHS), which interferes in the innovation dynamics of the national pharmaceutical sector, focus of the late national development plans. It was used the technique of science and technology data mining (tech mining) in a case study related to technologies of interest to the Ministry of Health, current and future, in the technological field of immunosuppressant drugs for solid organ transplantation. It was identified 48 patent applications apt to the priority examination, 21 future products in the fore mentioned technological field. It was also found that the lack of an explicit politics outlining the role played by the subsystem of pharmaceutical patents concession compromises the present political initiatives by means of which the recommendations to the public policy operators were elaborated.
\end{abstract}

Key Words: patent examination, technology prospection, pharmaceutical industry, tech mining. 


\title{
PROSPECÇÃO TECNOLÓGICA NA CONCESSÃO DE PATENTES NO BRASIL: estudo de caso em medicamentos imunossupressores de transplante de órgãos
}

Jussanã Cristina de Abreu ${ }^{1}$, Julia Paranhos ${ }^{2}$

\section{RESUMO}

O trabalho objetiva aplicar uma metodologia que visa subsidiar a operacionalização da priorização da análise técnica de pedidos de patentes pelo Instituto Nacional de Propriedade Industrial (INPI) e Agência Nacional de Vigilância Sanitária (Anvisa) baseada no instrumental teórico-prático de prospecção tecnológica. A priorização do exame de patentes é uma das linhas de gestão do backlog de patentes normatizada no INPI, com potencial de otimizar a morosidade da concessão de patentes farmacêuticas no Brasil e seus efeitos deletérios no Sistema Nacional de Inovação (SNI) e no Sistema Único de Saúde (SUS), influindo na dinâmica de inovação do setor farmacêutico nacional, foco dos recentes planos de desenvolvimento nacional. Foi utilizada a técnica de mineração de dados em ciência e tecnologia (tech mining) num estudo de caso em tecnologias de interesse do Ministério da Saúde (MS), atuais e futuras, no campo tecnológico dos medicamentos imunossupressores de transplante de órgãos sólidos. Foram identificados 48 pedidos de patentes aptos ao exame prioritário no país e 21 produtos futuros no campo tecnológico supracitado. Constatou-se ainda a ausência de uma política explicita que delineie o papel ocupado pelo subsistema de concessão de patentes farmacêuticas, compromete as iniciativas políticas vigentes pelo que foram tecidas recomendações aos operadores das políticas públicas no país.

Palavras-chaves: exame de patentes, prospecção tecnológica, indústria farmacêutica, mineração de dados em ciência e tecnologia.

\begin{abstract}
The objective of this paper is to apply a methodology that aims to aid the operationalization of prioritization of patent application technical analysis by INPI (National Institute of Intellectual Property) and Anvisa (Brazilian Health Regulatory Agency), based in the theoretical-practical technological prospection tools. The prioritization of patents examination is one of the lines of patents backlog management normatized by the Institute with potential to optimize the slowdown of pharmaceutical patents concession and its deleterious effects in the National Innovation System (NIS) and Brazilian Health System (BHS), which interferes in the innovation dynamics of the national pharmaceutical sector, focus of the late national development plans. It was used the technique of science and technology data mining (tech mining) in a case study related to technologies of interest to the Ministry of Health, current and future, in the technological field of immunosuppressant drugs for solid organ transplantation. It was identified 48 patent applications apt to the priority examination, 21 future products in the fore mentioned technological field. It was also found that the lack of an explicit politics outlining the role played by the subsystem of pharmaceutical patents concession compromises the present political initiatives by means of which the recommendations to the public policy operators were elaborated.
\end{abstract}

Key Words: patent examination, technology prospection, pharmaceutical industry, tech mining.

\section{INTRODUÇÃO}

Neste trabalho, centra-se no processo administrativo do subsistema de concessão das patentes de produtos e processos farmacêuticos no sistema de patentes brasileiro (SPB) relacionadas com tecnologias de interesse do MS, as últimas desde o ano de 2008 são publicadas e revisadas periodicamente no âmbito da Secretaria de Ciência, Tecnologia e Insumos Estratégicos daquele Ministério (SCTIE) ${ }^{3}$.

\footnotetext{
${ }^{1}$ Doutoranda, PPED/IE/UFRJ e Anvisa (Coordenação de propriedade intelectual). E-mail: jussana@gmail.com.

${ }^{2}$ Professora Adjunta, IE/UFRJ. Orientadora da tese. E-mail: juliaparanhos@ie.ufrj.br. 
A indústria farmacêutica relacionada com as supracitadas patentes tem sido alvo de várias políticas públicas $^{4}$ ligadas ao desenvolvimento nacional recente (Kupfer e Marques, 2013; Soares, Coronel e Filho, 2013), as quais tem por expectativa a geração de tecnologias passíveis de obtenção de patentes ao mesmo tempo em que incluem o acesso aos objetos de patentes no país. Estes fatos geram impactos no SUS tanto pela demanda crescente por produtos com maior conteúdo tecnológico junto ao MS como pelo contexto da base tecnológica nacional instalada ainda dependente de tecnologias exógenas.

O Sistema Brasileiro de Patentes (SPB) apresenta tendências dinâmicas e especificidades normativas onde se destacam, a existência de dois dispositivos na Lei nº9279/96 (LPI) que têm sido alvo de acalorados debates nos planos dos operadores de patentes e usuários dos seus respectivos produtos e processos a saber: o parágrafo único do art.40 e o artigo 229-C, os quais tratam de prazos de exclusividade de patentes e do subsistema de concessão de patentes farmacêuticas, respectivamente.

E o INPI, órgão executor de tal sistema, tem por desafio a gestão do backlog de patentes 5 influenciado pelo incremento de tais solicitações no país, além de sua capacidade de processamento.

Nesta linha, o parágrafo único do art.40 da LPI assegura ao titular da patente um período mínimo de dez anos de exclusividade, contados da data de concessão da patente. Desta forma, quando o processo administrativo de patentes demora mais de dez anos para ser decidido, há uma compensação proporcional ao atraso no prazo de exclusividade regular de vinte anos, o que prolonga a vigência da patente. Este dispositivo tem o potencial de criar prazos diferenciados da vigência de patentes no país, superiores ao disposto em TRIPS.

A frequente concessão de patentes farmacêuticas no escopo o parágrafo único do art.40 da LPI tem sido motivo de intenso debate no país. Isto porque, a LPI já contempla no art. 44, o direito de indenização ao titular da patente, nos casos de exploração indevida do seu objeto, no período entre a data de publicação do pedido e sua respectiva concessão (Barbosa, 2014a). Aqueles que apoiam a existência de computo diferenciado de prazo de exclusividade de patentes são os maiores usuários do SBP e, na indústria farmacêutica os grandes grupos empresariais multinacionais sediados no exterior (Cerqueira, Zucoloto e Souza, 2013) ocupam liderança. Para estes grupos a dinâmica da inovação e sustentabilidade de seus negócios pauta-se no uso estratégico e oportunista global das patentes (European Commission, 2008), sendo tal dispositivo legal um importante instrumento estratégico.

No entanto, sua aplicação para quase totalidade de patentes farmacêuticas concedidas no Brasil, em razão da morosidade do processo de concessão de tais patentes, acarreta efeitos nos preços dos produtos com elas associados com desdobramentos no SUS (Jannuzzi e Vasconcellos, 2013). Ao criar barreiras adicionais à entrada de novos competidores este artigo acaba por limitar a oferta de produtos alternativos e a dinâmica de inovação nas trajetórias tecnológicas objeto de tais patentes ${ }^{6}$.

Por sua vez, o artigo 229-C da LPI dentre outras questões, disciplina a necessidade de participação da Anvisa na avaliação de patentes de produtos e processos farmacêuticos (anuência prévia) juntamente ao INPI, criando, desta forma, um subsistema de concessão de patentes no setor farmacêutico. Este subsistema tem sido alvo de uma trajetória de altos e baixos em relação ao apoio a sua existência onde aqueles que o defendem argumentam que o mesmo é uma inovação regulatória em prol do incremento da qualidade das patentes a ser seguido por demais países em desenvolvimento (Walls, Smith e Drahos, 2015), traz contribuições para o acesso aos medicamentos e favorece a saúde pública (Guimarães e Corrêa, 2012), sendo plenamente compatível com o sistema internacional de propriedade industrial do

\footnotetext{
${ }^{4}$ Com destaque para as seguintes ações: Política Nacional de Ciência, Tecnologia e Inovação em Saúde (PNCTIS), a Política Tecnológica e de Comércio Exterior (PITCE) - 2004-2007-, Plano de Desenvolvimento Produtivo (PDP) - 2008-2010-e, Plano Brasil Maior (2011).

${ }^{5}$ Pedidos de patentes aguardando decisão sobre a sua validade.

${ }^{6}$ Diante de tais efeitos a Associação Brasileira das Indústrias de Química Fina, Biotecnologia e suas Especialidades (ABIFINA) ajuizou, em 4 de novembro de 2013, uma Ação de Inconstitucionalidade (ADI) n5061 pela qual busca eliminar o parágrafo único do art. 40 da LPI, o qual aguarda concluso no Supremo Tribunal Federal (ABIFINA, 2013).
} 
The Agreement on Trade-Related Aspects of Intellectual Property Rights ou Acordo TRIPS (Barbosa, 2014b; Plaza, 2012).

Já os opositores ao subsistema vigente sustentam que este onera negativamente os requerentes de patentes, gera insegurança jurídica em razão da participação de dois entes no processo de obtenção de patentes (Avila, 2014), cria requisito não previsto no sistema internacional de patentes (Guimarães e Corrêa, 2012), ressaltam que as patentes servem ao desenvolvimento tecnológico e comercial do país onde o INPI é a única autarquia legalmente constituída para a sua regulação no país (Tojal e Pessôa, 2008). No entanto, parte dos opositores são favoráveis ao entendimento que a participação da Anvisa no SBP deve ser sugestiva no que tange as normas de patenteabilidade e estritamente voltada para aspectos de segurança de produtos e processos em saúde pública (Mueller e Taketsuma Costa, 2014), o que é compatível com o entendimento exarado pela Advocacia Geral da União, AGU (AGU, 2009).

A polarização de apoio ao subsistema de concessão de patentes inflama-se por sua institucionalização infralegal incompleta e pela inexistência de correspondência de organização semelhante no plano internacional e, acima de tudo, pela existência de discordância quanto ao atendimento às condições de patenteabilidade ${ }^{7}$. Estes fatos produziram alterações na operacionalização do subsistema, em tela, sugeridas por um grupo de trabalho interministerial (Grupo de Trabalho Interministerial, 2012), após provocação do INPI à Advocacia Geral da União (AGU), a última publicou parecer opinativo, ratificado pelo Advogado Geral da União, que blinda a ação da Anvisa ao exame de segurança e eficácia em patentes (AGU, 2009).

Os entendimentos da AGU e do Grupo Interministerial não foram capazes de equalizar os dilemas da anuência prévia de patentes quanto ao escopo decisório de cada ente do governo arrolado no processo de concessão das patentes farmacêuticas disposto no artigo 229-C da LPI, resultando no deslocamento de parte das decisões, do plano administrativo para o judiciário, além da ocorrência de outras situações no plano administrativo do processo de concessão de patentes a saber: não finalização administrativa de parte dos pleitos avaliados na Anvisa - quando a atuação da Agência nos processos de patentes provoca modificações fundamentadas em condições de patenteabilidade da LPI -, pelo INPI, não conclusão de pedidos de patentes avaliados previamente ao Grupo de Trabalho Interministerial onde houve discordância de opinião em patenteabilidade entre INPI e Anvisa e, processos de patentes finalizados sem passar por nenhuma das situações anteriormente descritas. Desta forma, uma parcela dos pedidos de patentes sujeitos ao art.229-C da LPI estão submetidos a dilatação do tempo decisório adicional nos termos do parágrafo único do art. 40 da mesma legislação pela ausência de ação jurídica com poder vinculante às diretorias do INPI e Anvisa.

No espectro recente da anuência prévia de patentes destaca-se o trâmite de uma ação coletiva judicial interposta pela Interfarma ${ }^{8}$ junto a $16^{\mathrm{a}}$ vara da seção judiciária do Distrito Federal, Processo $\mathrm{n}^{\mathrm{o}}$ 8740943.2014.4.01.3400, de 21 de novembro de 2014, que tem por foco anular parcialmente a aplicação da resolução de anuência prévia da Anvisa relacionada com o exame de condições de patenteabilidade (Melo e PRF 1ª Região, 2015) ainda sem conclusão.

Adicionalmente, o MS publicou a Portaria nº736/2014 que sinaliza à Anvisa a necessidade do exame das condições de patenteabilidade da LPI nos pedidos de patentes que tenham por objeto: antivirais e antirretrovirais, doenças negligenciadas, doenças degenerativas (Alzheimer/ Parkinson), imunossupressores, doenças mentais (antipsicóticos/ anticonvulsivantes), produtos obtidos por rotas biológicas, vacinas e soros, hemoderivados e, produtos oncológicos. Este fato demonstra explicito interesse daquele Ministério em participar da concessão de patentes, por meio da Anvisa, resguardando o poder de veto daquela autarquia para atuar nos pedidos de patentes com fundamentos de patenteabilidade para as tecnologias consideradas mais estratégicas para resolução de problemas de saúde e

\footnotetext{
${ }^{7}$ Para uma parcela reduzida dos documentos de patentes avaliados em tal sistema desde sua implantação em 2001, entre as organizações envolvidas (Guimarães e Corrêa, 2012).

${ }^{8}$ Associação da Indústria Farmacêutica de Pesquisa - Interfarma.
} 
desenvolvimento econômico e social pois seu texto faz eco as demais regras que sinalizam os produtos estratégicos para o Complexo Econômico e Industrial da Saúde (CEIS) e às políticas de medicamentos e assistência farmacêutica do SUS (Ministério da Saúde e Gabinete do Ministro, 2014a).

Finalmente, a existência do backlog de patentes no SBP, dilata o tempo de decisão sobre a validade das patentes requeridas, pelo acúmulo de processos aguardando decisão e, adicionalmente, tem potencial de afetar a qualidade decisória em pedidos de patentes no país, gerando custos de transação, excessivos, que comprometem o desenvolvimento tecnológico futuro, alternativo ou reprodutivo de produtos objetos de documentos de patentes aguardando decisão.

Neste sentido, as políticas públicas de medicamentos e assistência farmacêutica do SUS são as mais afetadas quando os pedidos de patentes com elas relacionados restam pendentes de decisão, pois as incertezas sobre seus respectivos mérito e escopo de proteção caso concedidos inibe a entrada de novos participantes, afetando a concorrência e a dinâmica de inovação do setor. Assim, o papel do SPB nos planos de desenvolvimento nacional, onde o setor farmacêutico ocupa importante posição, é posto em cheque num contexto onde obter decisão sobre tais patentes dura mais de dez anos (Câmara, 2015) ou resta inconclusa na administração pública pela discordância entre as autarquias federais envolvidas.

A implementação de exame prioritário de pedidos de patentes de tecnologias-chaves para o Brasil, dentre as quais aqueles relacionados com o subsistema de patentes farmacêuticas supracitado, é uma alternativa de gestão do backlog, reconhecida e implementada internacionalmente (Musskopf, 2012). Tal mecanismo, foi normatizado no INPI, pela Portaria $n^{\circ} 80 / 2013$, a prerrogativa para que o MS requeira ao INPI a sua operacionalização para os produtos estratégicos para o SUS, incluindo aqueles do subsistema da anuência prévia, onde sua plena efetivação demanda instrumental teórico-metodológico para identificar os pedidos de patentes relacionados com as mesmas, na qual as ferramentas de prospecção tecnológica assume papel central.

A prospecção tecnológica é um segmento dos estudos de futuro, originado no pós-guerra, que envolve um processo de investigação alinhado à seara da ciência, tecnologia e inovação com vistas subsidiar tomadores de decisões com insumos informacionais capazes de influenciar políticas públicas originado no pós-guerra (Coates, 1985; Godet, 1982).

Os estudos de prospecção tecnológica contam com instrumental dinâmico e flexível onde se destaca a técnica de mineração tecnológica em ciência e tecnologia (tech mining). Esta técnica consiste em analisar informações das bases de dados de ciência e tecnologia, patentes e demais publicações não patentárias e compõe-se de três fases: planejamento (identificação do tema, seleção das fontes de informações e estratégia de busca para a obtenção de dados), implementação (limpeza e organização dos dados e análise) e informe de resultados (Porter e Cunningham, 2004).

Desta forma, neste trabalho, com o intuito de explicitar o potencial de uso desta técnica, aplica-se uma metodologia de prospecção baseada em tech mining para identificar pedidos de patentes relacionados com o campo tecnológico dos medicamentos imunossupressores para uso no pós-transplante de órgãos sólidos ${ }^{9}$ depositados no Brasil, atuais e futuros. Objetiva-se subsidiar a operacionalização da priorização de sua respectiva análise técnica pelas organizações diretamente envolvidas (INPI e Anvisa) prevista na Portaria INPI n80/2013 e, colaborar com a otimização do subsistema de patentes farmacêuticas no país, elemento-chave para a dinâmica de inovação do setor farmacêutico.

A escolha do supracitado campo tecnológico reside no fato do mesmo pertencer ao escopo do subsistema de análise de patentes, em tela, estar incluso nas políticas de medicamentos, assistência farmacêutica e

\footnotetext{
${ }^{9}$ O termo transplante é definido pela OMS como a transferência (enxerto) de células humanas, tecidos ou órgãos de um doador para um recipiente com o propósito de reestabelecer o funcionamento do corpo (WHO, 2016), onde neste trabalho são considerados órgãos sólidos, rim, fígado, coração e pulmão.
} 
parcerias de desenvolvimento produtivo ${ }^{10}$. Além disso, é insumo do Sistema Nacional de Transplantes de Órgãos (SNT), o maior programa público desta natureza em nível mundial, que desde o ano de 1997, tem apresentado desempenho crescente em aportes financeiros governamentais, número de procedimentos executados e número de cadastro de intenção para doação de órgãos pós-morte manifestados em redes sociais (Ministério da Saúde e Rabelo, 2012).

\section{METODOLOGIA}

A presente metodologia faz uso de mais de uma base de dados de informações tecnológicas (patentárias e não patentárias) sendo composta por duas rotas (A e B) procedimentais, utilizando parâmetros operacionais customizados ao supracitado campo tecnológico onde a primeira não utiliza designações diretamente relacionadas com produtos como é feito na segunda.

O uso de mais de uma rota procedimental objetiva a identificação do maior número de patentes relacionadas com produtos já comercializados e futuros (em fase de pesquisa clínica). Estas rotas, e as respectivas bases de informações consultadas estão subsequentemente particularizadas, destacando-se que as consultas as bases de dados ocorreram entre dezembro de 2015 e fevereiro de 2016.

\subsection{Metodologia de prospecção tecnológica: rota A}

Esta rota compõe-se de duas rotas que diferem entre si pelo uso do software Vantagepoint para o processamento automático e semi-automático para limpeza e organização das informações obtidas.

\subsubsection{Estratégia A1}

a) buscar documentos de patentes na base Thomson Innovation utilizando os argumentos de busca textual, inseridos em inglês, no campo de entrada Text fields All - DWPI combinados entre si com operadores lógicos para localização do conjunto de documentos de patentes preliminar,

Os argumentos de busca ${ }^{11}$ utilizados foram: $\{$ immunosuppress* AND (agent* $\boldsymbol{O R}$ drug) AND (transplant* OR allograft) AND human* AND(graft OR solid organ) AND rejection\} AND \{immunosuppress* AND (agent* OR drug) AND (transplant* OR allograft) AND human*AND (graft OR organ) AND rejection\}.

b) refinar os resultados obtidos na etapa anterior utilizando argumentos de filtro no campo IPC-Any DWPI, da base em tela, baseados nas Classificação Internacional de Patentes (CIP) constantes do conjunto de documentos de patentes da etapa anterior,

Os documentos de patentes que se vinculem com CIP que não estejam relacionadas ao escopo da anuência prévia de patentes da Anvisa são eliminados, os demais são mantidos.

c) refinar os documentos de patentes remanescentes com a operacionalização de sub-buscas sequenciais na base Thomson Innovation utilizando os campos de entrada Abstract - Activity e Abstract - use, alimentados com argumentos textuais para obter o conjunto de documentos de patente inicial,

d) exportar os resultados da etapa anterior para o software Vantagepoint para limpeza do campo Abstract mechanism of action (NLP) (Phrases) ou resumo de mecanismo de ação processado por linguística computacional em frases, gerado pelo mesmo a partir do processamento do campo Abstract - mechanism of action da base Thomson Innovation, incluindo a importação do filtro de análise Thomson Innovation (Patents) para o software, e, de todos os dados informacionais dela disponíveis associados com as famílias de patentes no formato Thomson Data Analyzer export format, executar limpeza automática de tal campo.

e) executar limpeza e aplicação de filtro adicional (semi-automáticos) no campo Abstract mechanism

\footnotetext{
${ }^{10}$ Alguns produtos imunossupressores para transplante de órgãos sólidos já foram ou estão nas listas de PDP que buscam uma articulação entre parceiros públicos e privados para a produção, transferência de tecnologia, inovação e redução da vulnerabilidade do SUS como p.ex. tacrolimo, sirolimo, everolimo, micofenolato de sódio (Ministério da Saúde, 2009, 2016).

${ }^{11}$ Os operadores lógicos AND e OR correspondem em português a "e” e “ou”.
} 
of action (NLP) (Phrases) para a obtenção do conjunto de documentos de patentes intermediário.

São considerados aptos a formar o conjunto de documentos de patentes intermediário os documentos de patentes que estejam relacionados com mecanismos de ação farmacológica genéricos presentes no campo supracitado, localizados durante a limpeza do mesmo, sendo que os demais documentos são eliminados.

f) aplicar filtro automático no campo Family Member - Numbers, no software Vantagepoint, o qual exibe as identificações alfanuméricas de todos os documentos de patentes do conjunto de documentos de patentes obtidos na etapa anterior, para obter o conjunto de documentos de patentes depositados no Brasil.

O conjunto de documentos de patentes depositados no Brasil é formado pelos documentos de patentes membros das famílias de documentos de patentes que possuam o radical BR na identificação alfanumérica dos mesmos.

g) identificar, a partir do conjunto de documentos de patentes obtidos na etapa anterior, aqueles considerados aptos ao exame prioritário de patentes no Brasil.

São considerados aptos ao exame prioritário no Brasil os documentos de patentes que não estejam nas situações arquivado, desistência homologada, patente extinta, patente concedida, exigência, fase de recurso, exame prioritário ou em processo judicial na base de patentes do INPI, na base de patentes do INPI, e, concomitante, estejam relacionados a medicamento (em comercialização ou desenvolvimento) imunossupressor para uso no pós-transplante de órgãos sólidos na base Cortellis for Competitive Intelligence, os quais formam a lista de documentos de patentes aptos ao exame prioritário, os demais serão eliminados.

A constatação de relação entre pedido de patente e medicamento é realizada pela verificação das informações Companies and drugs exibida pela base Cortellis for Competitive Intelligence após consulta por dados identificadores de um pedido de patente.

\subsubsection{Estratégia A2}

a) buscar documentos de patentes na base Thomson Innovation utilizando os campos de entrada Abstract-Activity-DWPI, Abstract - Use-DWPI - com uso de argumentos de busca textual em inglês - e, IPC - Any (alfanumérico), associados entre si com operadores lógicos, para a obtenção do conjunto de documentos de patentes inicial,

b) configurar a base de dados para exibir todos os membros das famílias do conjunto de documentos inicial e aplicar filtro no campo Country code calibrado para a sigla BR, para obter aqueles com elas vinculados solicitados no Brasil os quais formam o conjunto de documentos de patentes intermediário,

c) avaliar os títulos, resumos e CIP dos documentos de patentes obtidos na etapa anterior onde aqueles considerados pertinentes formam o conjunto de documentos de patentes depositado no Brasil,

São considerados pertinentes aqueles documentos de patentes que estejam no escopo de análise de anuência prévia de patentes da Anvisa.

d) identificar, a partir do conjunto de documentos de patentes obtidos na etapa anterior, aqueles considerados aptos ao exame prioritário de patentes no Brasil,

São considerados aptos ao exame prioritário no Brasil os documentos de patentes que não estejam nas situações arquivado, desistência homologada, patente extinta, patente concedida, exigência, fase de recurso, exame prioritário ou em processo judicial na base de patentes do INPI, e, concomitante, estejam relacionados a medicamento (em comercialização ou desenvolvimento) imunossupressor para uso no póstransplante de órgãos sólidos na base Cortellis for Competitive Intelligence, os quais formam a lista de 
documentos de patentes aptos ao exame prioritário, os demais serão eliminados.

A constatação da relação do pedido de patente com medicamento imunossupressor para transplante, como anteriormente citado, é realizada pela verificação das informações Drugs and deals exibida pela base Cortellis for Competitive Intelligence após consulta por dados identificadores do pedido de patente.

\subsubsection{Metodologia de prospecção tecnológica: rota B}

Esta rota compõe-se de cinco etapas onde as duas primeiras são buscas preliminares, operacionalizadas com a finalidade de identificar as designações dos insumos ativos aprovados para uso em transplante de órgãos sólidos pelo MS e aqueles que estão em avaliação clínica em nível global (futuros), respectivamente, na data de consulta como discriminado nas alíneas abaixo, que servem de insumo informacional para a etapa subsequente.

a) buscar no sítio eletrônico do MS pelos medicamentos imunossupressores inclusos no Protocolo Clínico e Diretrizes Terapêuticas (PCDT) de imunossupressão em transplante de órgãos sólidos, bem como na lista Rename por medicamentos adicionais vinculados com mesma indicação terapêutica, seguida pela compilação dos respectivos insumos ativos com eles vinculados.

b) buscar na base ClinicalTrials.gov por testes clínicos relacionados com medicamentos (em comercialização ou em fase experimental) para uso terapêutico no pós-transplante de órgãos sólidos utilizando argumentos de busca, textuais e pré-estabelecidos, nos campos de entrada search terms (termos de busca), recruitment (estágio de recrutamento de voluntários), condition (condição), study status (situação do estudo), study results (resultados do estudo) e, study type (tipo de estudo) seguida pela identificação daqueles considerados pertinentes, compilando os respectivos insumos ativos com estes vinculados.

O teste clínico é considerado pertinente após leitura de seu respectivo título, resumo e demais dados onde aqueles relacionados com terapia celular, radiação, procedimento cirúrgico de transplante, condicionamento pré-transplante, ou voltados para avaliar o desempenho de imunossupressores em terapias de outras patologias (p.x. câncer, diabetes) são excluídos e os remanescentes tem seus respectivos insumos ativos catalogados.

Os insumos ativos vinculados aos testes clínicos considerados relevantes são identificados no campo intervention (intervenção) de seu respectivo resumo.

c) elaborar um quadro sintetizando informações dos insumos ativos de medicamentos imunossupressores para uso no pós-transplante de órgãos sólidos oriundos do PCDT, Rename e Clinical Trials.

d) buscar na base Cortellis Competitive Intelligence por documentos de patentes associados com os insumos ativos dos medicamentos localizados na etapa anterior utilizando a interface Advanced Search - Patents e o campo Drug (no menu suspenso), onde deve ser inserida a designação de cada insumo ativo, em inglês. Esta etapa será realizada isoladamente para cada insumo ativo, assim como, as posteriores.

e) refinar os resultados de documentos de patentes obtido, com o acionamento de filtro automático da base, em tela, aplicado no campo tipo de documentos de patentes (patent type) onde o mesmo é configurado para que sejam exibidos os resultados apenas daqueles documentos que estejam voltados para formulação (formulation), combinação de drogas (drug combination), novo uso (new use), processo (process) e produto (product), os quais tratam do escopo da anuência prévia de patentes da Anvisa, para formar o conjunto de documentos de patente inicial.

f) configurar a base de dados para exibir todos os membros das famílias de documentos de patentes que formam o conjunto inicial de documentos de patentes e implementar o filtro automático no campo país de publicação (publication coutry) calibrado para Brasil para identificar neste conjunto de documentos aqueles que geraram depósitos no Brasil, os quais formam tanto o conjunto de 
documentos de patente intermediário como o conjunto de documentos de patentes depositados no Brasil.

g) consultar na base de patentes do INPI a situação dos documentos de patentes obtidos na etapa anterior e avaliar quais são considerados aptos ao exame prioritário de patentes no Brasil.

São considerados aptos ao exame prioritário no Brasil os documentos de patentes que não estejam nas situações arquivado, desistência homologada, patente extinta, patente concedida, exigência, fase de recurso, exame prioritário ou em processo judicial na base de patentes do INPI, na base de patentes do INPI, e, concomitante, estejam relacionados a medicamento (em comercialização ou desenvolvimento) imunossupressor para uso no pós-transplante de órgãos sólidos na base Cortellis for Competitive Intelligence, os quais formam a lista de documentos de patentes aptos ao exame prioritário, os demais serão eliminados.

h) elaborar um quadro sintetizando as informações relacionadas com os documentos de patentes considerados aptos ao exame prioritário de patentes no Brasil incluindo: número do documento de patente, insumos ativos dos medicamentos imunossupressores, objeto de maior escopo requerido, situação no INPI, data da situação no INPI associada com os mesmos.

Após a implementação e obtenção dos resultados das rotas A e B da metodologia de prospecção tecnológica, compilar os resultados obtidos e, realizar verificação no sítio eletrônico da Coopi/Anvisa (Anvisa, 2016b) para aferir a situação daqueles que estejam na situação de anuência prévia, no sítio do INPI, para a obtenção da lista de pedidos de patentes aptos ao exame prioritário, que será composta apenas por pedidos que não tenham passado por decisão da Agência até a data, a qual organiza-se pela hierarquia de objeto requerido de maior relevância para cada produto identificado.

Finalmente, todos os insumos ativos de documentos de patentes identificados pela aplicação da metodologia de prospecção tecnológica foram compilados, sendo exibidos num Quadro como alternativas futuras neste campo tecnológico aqueles que não estejam registrados no país, aferidos pela consulta ao sítio da Anvisa (Anvisa, 2016a) ou identificados como em fase clínica para uso no pós-transplante de órgãos pela aplicação da supracitada metodologia.

\section{RESULTADOS E DISCUSSÕES}

A partir da aplicação das rotas de prospecção tecnológica A e B foram localizadas 4.511 famílias de documentos de patentes ${ }^{12}$, as quais resultaram em apenas 466 depósitos no Brasil, tendo sido eliminados 418 do último para a formação da lista de candidatos ao exame prioritário de patentes no Brasil, por já terem passado por andamento/decisão no processo administrativo das autarquias diretamente envolvidas. Os documentos de patentes remanescentes (48) formam a lista de pedidos candidatos ao exame prioritário do Quadro 1.

\footnotetext{
12 Uma família de documentos de patentes neste trabalho é definida por um conjunto de documentos de patentes que possui pelo menos um número de prioridade em comum.
} 
Quadro 1: Lista de pedidos de patentes candidatos ao exame prioritário no campo dos imunossupressores para o uso pós transplante de órgãos sólidos: identificação (ID), documento ( $\mathrm{N}^{\circ}$ ), medicamento, objeto de maior escopo requerido.

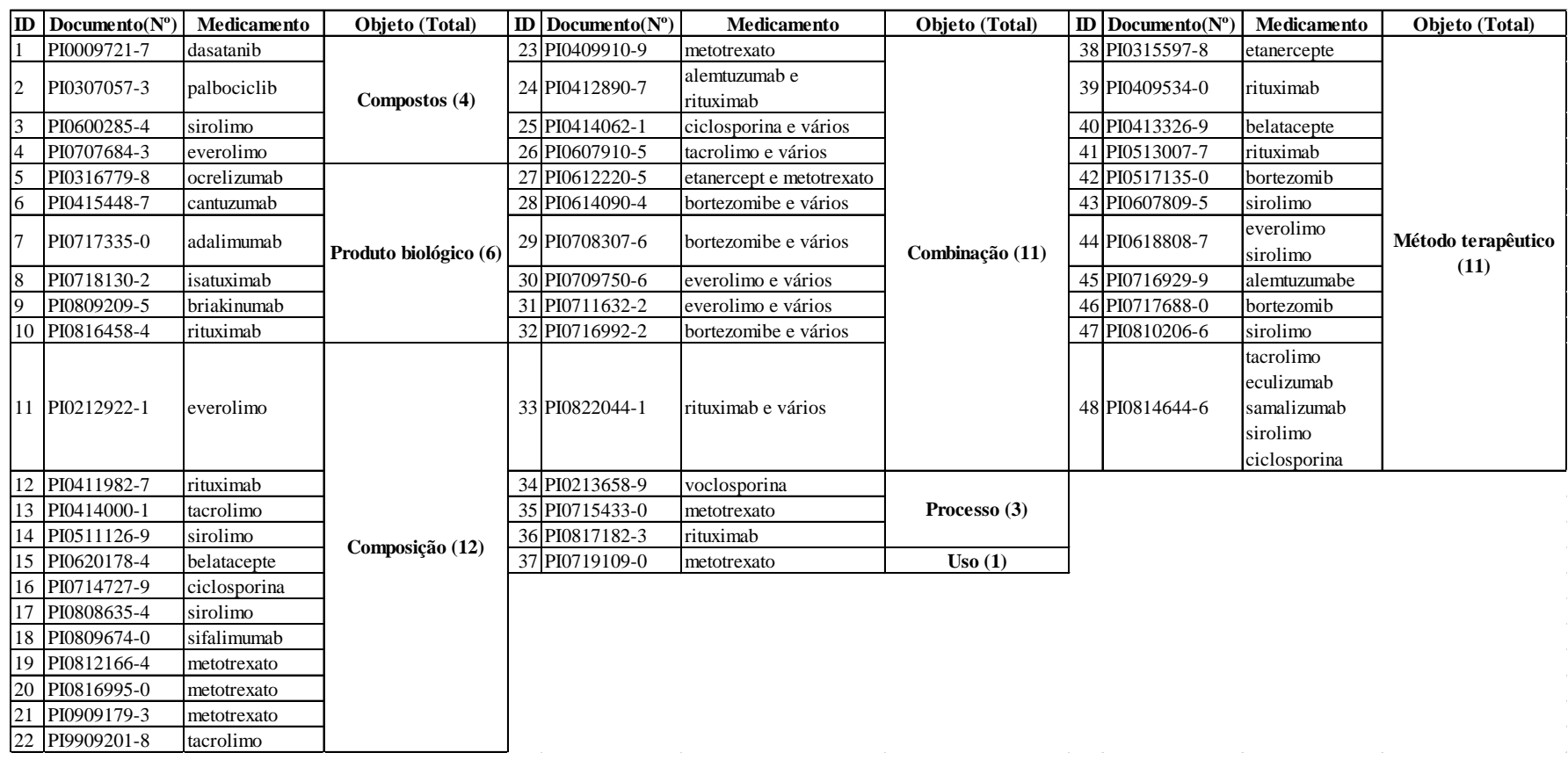

Fonte: Elaboração própria.

Nota: Nos documentos de objeto combinação foram explicitados apenas os componentes da classe terapêutica imunossupressor.

O Quadro 1 ilustra que a maior parte dos documentos de patentes candidatos ao exame prioritário têm por objeto de maior escopo requerido, composição ou combinação farmacêuticas seguidos por produto biológico e composto e apenas um pedido de patente relacionado com processo.

Ademais, destaca-se a identificação de um grupo de documentos de patentes no Quadro 1 que tem por objeto principal método terapêutico (10), o qual não é privilegiável por não ser considerado invenção pela LPI.

Finalmente, constata-se que 23 pedidos de patentes no conjunto dos documentos de patentes do Quadro 1 têm ano de depósito inferior a 2006, os quais não sendo decididos com celeridade gozarão da prerrogativa de compensação do prazo de exclusividade do parágrafo único do art. 40 da LPI.

Os medicamentos futuros identificados na metodologia utilizada estão elencados no Quadro 2.

Quadro 2: Futuros IFAs para uso no pós-transplante de órgãos sólidos identificados pela metodologia de prospecção tecnológica e respectiva rota de obtenção destas.

\begin{tabular}{|c|c|c|c|c|c|}
\hline $\mathrm{N}^{\mathbf{0}}$ & IFA & \multirow{15}{*}{$\begin{array}{c}\text { Rota } \\
\text { biológica }\end{array}$} & $\mathbf{N}^{\mathbf{0}}$ & IFA & \multirow{8}{*}{ Quimica } \\
\hline 1 & adalimumab & & 15 & bortezomibe & \\
\hline 2 & alefacepte & & 16 & dasatanibe & \\
\hline 3 & alemtuzumab & & 17 & palbociclibe & \\
\hline 4 & belatacepte & & 18 & pentostatina & \\
\hline 5 & briakinumab & & 19 & regorafenibe & \\
\hline 6 & cantuzumab & & 20 & sorafenibe & \\
\hline 7 & daclizumab & & 21 & tofacitinibe & \\
\hline 8 & eculizumab & & & & \\
\hline 9 & etanercepte & & & & \\
\hline 10 & isatuximab & & & & \\
\hline 11 & ocrelizumab & & & & \\
\hline 12 & rituximab & & & & \\
\hline 13 & samalizumab & & & & \\
\hline 14 & sifalimumab & & & & \\
\hline
\end{tabular}

Fonte: Elaboração própria a partir da Rename (MS, SCTIE e DAF, 2015) e da base ClinicalTrials.gov. 
Legenda: IFA refere-se ao insumo farmaceuticamente ativo.

A partir do Quadro 2 nota-se que 67\% dos medicamentos futuros do campo tecnológico estudado são produtos obtidos por rota biotecnológica, onde a maior parte são anticorpos monoclonais ou proteínas de fusão e, os demais por rota química.

\section{CONCLUSÃO}

Dados os desafios enfrentados pelo SBP relacionados tanto com o incremento de pedidos de patentes nos últimos anos como pelas especificidades de sua institucionalização conclui-se a partir deste trabalho, que o uso da prospecção tecnológica pode dar agilidade e maior efetividade ao uso da priorização da análise de pedidos de patentes de interesse do MS.

A aplicação da metodologia de prospecção tecnológica permitiu localizar 48 pedidos de patentes aptos a solicitação do exame prioritário pelo MS ao INPI e 21 produtos(medicamentos) futuros a serem utilizados no pós-transplante de órgãos, insumos do SNT cujo sucesso depende da integralidade do acesso a tais produtos, pelos que deles necessitam, e, alvo de políticas públicas para a internalização de sua cadeia de produção e fomento da diversificação da cadeia tecnológica aqui instalada dependente de exportações.

O uso do exame prioritário de patentes pelo MS junto ao INPI tem o potencial de atuar como elemento estratégico para as iniciativas de políticas públicas vigentes de saúde e desenvolvimento, uma vez que a maioria dos pedidos de patentes identificados neste trabalho, já está inclusa no dispositivo legal de compensação extra ao prazo de exclusividade de 20 anos regular da LPI.

Desta forma, a morosidade do SBP onera tanto os operadores do SBP como os usuários dos produtos objetos de patentes, afetando não apenas a dinâmica do SNI nacional, mas também, comprometendo, no campo tecnológico do presente trabalho. o sucesso do Sistema Nacional de Transplantes no que tange ao acesso aos medicamentos atuais e futuros pelos que deles necessitam, (medicamentos objetos de patentes têm preço superior na ausência de alternativas no mercado). Ao mesmo tempo, os contratos de PDP podem incluir produtos objeto de patentes que poderiam ser decididas previamente a sua pactuação para minimizar custos de transação envolvidos no processo de transferência de tecnologia.

Diante dos resultados obtidos, recomenda-se aos operadores de políticas públicas a adesão ao uso da prospecção tecnológica, em sua vertente de mineração de dados em ciência e tecnologia, para dinamizar a o uso do exame prioritário de patentes, entendido como instrumento explícito de suporte às demais iniciativas de políticas públicas de desenvolvimento do setor farmacêutico nacional e sucesso do SUS.

\section{REFERÊNCIAS}

ABIFINA. Ação Direta de Inconstitucionalidade n⿳05061. Institucional. Disponível em: $<$ http://www.stf.jus.br/portal/peticaoInicial/verPeticaoInicial.asp?base=ADIN\&s1=patente\&processo=50 61>. Acesso em: 8 ago. 2015.

AGU. Parecer $\mathbf{n}^{0}$ 210/PGF/AE/2009. Conflito positivo de atribuições. INPI e ANVISA. Finalidade institucional. Patente. Anuência prévia. Poderes administrativos implícitos. Inexistência. Princípios da legalidade, especialidade e ato tutela. Alcance e aplicação dos artigos 229-C e 230, introduzidos na Lei n⿳9279, de 14.5.1996, pela Lei $n^{0} 10.196$, de 2001. Manifestação conclusiva da ConsultoriaGeral da União. Ação anulatória. Brasília: AGU, 16 out. 2009.

ANVISA. Listas de preços de medicamentos. Institucional. Disponível em: $<$ http://s.anvisa.gov.br/wps/s/r/bs>. Acesso em: 24 mar. 2016a.

Regularização de Produtos - Propriedade Intelectual. Institucional. Disponível em:

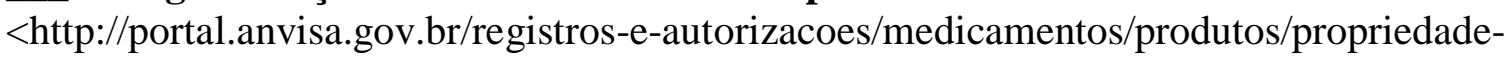
intelectual/relatorios-de-informacao>. Acesso em: 26 jun. 2016b.

AVILA, J. Intellectual property on life sciences and biotechnology: state of affairs, impacts and perspectives. BMC Proceedings, v. 8, n. Suppl 4, p. O48, 1 out. 2014. 
BARBOSA, D. B. Desapertanto em cima do mercado: A prorrogação de patentes do art. 40 parágrafo único do CPI/96. Apresentação Academia de Propriedade Industrial do INPI apresentado em Seminário Patentes: Inovação em prol da competitividade nacional. Rio de Janeiro, 10 abr. 2014a. Disponível em: <http://goo.gl/4b77ik>. Acesso em: 27 jul. 2015.

Anuência prévia pela ANVISA: só para proteger a saúde pública? Direito Contemporâneo e Constituição, v. 7, n. 1, p. 50-107, 24 out. 2014b.

CÂMARA, V. B. Backlog do INPI: custos e oportunidades. Apresentação apresentado em Comissão de Relações Exteriores e de Defesa Nacional - Audiência Pública. Câmara dos Deputados - Brasília, 9 jun. 2015. Acesso em: 8 jan. 2015

CERQUEIRA, L.; ZUCOLOTO, G. F.; SOUZA, A. DE M. A revisão da Lei de patentes: inovação em prol da competitividade nacional. 1. ed. Brasília: Câmara dos Deputados/ Edições Câmara, 2013. v. 1

COATES, J. Foresight in federal government policy making. Futures Research Quartely, v. 1, p. 29-53, 1985.

EUROPEAN COMMISSION. Pharmaceutical sector inquiry: preliminary report (DG Competition Staff Working Paper). Institucional. Disponível em: $<$ http://ec.europa.eu/competition/sectors/pharmaceuticals/inquiry/preliminary_report.pdf >. Acesso em: 20 jun. 2016.

GODET, M. From forecasting to "la prospective"a new way of looking at Futures. Journal of Forecasting, v. 1, n. 3, p. 293-301, 1982.

GRUPO DE TRABALHO INTERMINISTERIAL. Relatório de Análise e Sugestão de Critérios, Mecanismos, Procedimentos, Obrigações e Possíveis Instrumentos Formais para Articulação entre a ANVISA e o INPI com Vistas à Execução do Art. 229-C da Lei n09279/1996. Brasilia: [s.n.]. Disponível em: <http://migre.me/eQlLq>.

GUIMARÃES, E.; CORRÊA, M. Propriedade intelectual e saúde pública: o papel da Agência Nacional de Vigilância Sanitária no patenteamento farmacêutico no Brasil. Revista Eletrônica de Comunicação, Informação \& Inovação em Saúde, v. 6, n. 3, 2012.

JANNUZZI, A. H. L.; VASCONCELLOS, A. G. Um estudo sobre a concessão de patentes de medicamentos no Brasil e suas implicações para a continuidade do êxito na política de medicamentos genéricos: Um estudo sobre a concessão de patentes de medicamentos no Brasil e suas implicações para a continuidade do êxito na política de medicamentos genéricos. Anais. In: ALTEC 2013: Políticas de Gestão de Ciência e Tecnologia nos Espaços Latino-Iberoamericanos. Portugal (Porto): Centro de Estudos em Inovação, Tecnologia e Políticas de Deenvolvimento, IN+, do Instituto Superior Técnico, $\quad$ out. 2013Disponível em: <http://www.altec2013.org/docs/PROCEEDINGS_ALTEC2013_v3.pdf>

KUPFER, D.; MARQUES, F. S. The Return of Industrial Policy in Brazil. In: STIGLITZ, J. E.; YIFU, J. L. (Eds.). The Industrial Policy Revolution I: The Role of the government beyond ideology. IEA Industrial Policy Roundtable. England: Palgrave Macmillan, 2013. v. 1p. 327-339.

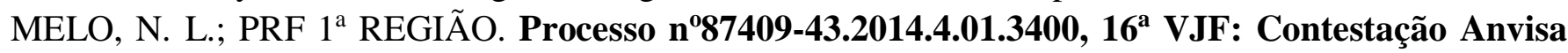
(Ré), 27 abr. 2015. Acesso em: 4 jan. 2016.

MINISTÉRIO DA SAÚDE. Planilha das Parcerias de Desenvolvimento Produtivo (PDP), 2009.

. Propostas de projetos de parcerias para o desenvolvimento produtivo (PDP) aprovadas de 2009 a 2014: DECIT/SCTIE. Portal. Disponível em: <http://goo.gl/73m3SI>. Acesso em: 10 jul. 2015. MINISTÉRIO DA SAÚDE; GABINETE DO MINISTRO. Portaria nº 3089, de 11 de dezembro de 2013. Redefine a lista de produtos estratégicos para o Sistema Único de Saúde (SUS) e as respectivas regras e critérios para sua definição. DOU. 13 dez. 2013, Sec. 1, p. 153.

Portaria $n^{0}$ 736, de 2 de maio de 2014. Define, para fins do exame de prévia anuência da Agência Nacional de Vigilância Sanitária (ANVISA), conforme o art. 229-C da Lei $n^{0}$ 9.279, de 1996, os produtos ou processos farmacêuticos considerados de interesse para as políticas de medicamentos ou de assistência farmacêutica no âmbito do SUS. DOU. 5 maio 2014a, Sec. 1, p. 38. 
. Portaria $\mathrm{n}^{0}$ 2531, de 12 de novembro de 2014. Redefine as diretrizes e os critérios para a definição da lista de produtos estratégicos para o Sistema Único de Saúde (SUS) e o estabelecimento das Parcerias para o Desenvolvimento Produtivo (PDP) e disciplina os respectivos processos de submissão, instrução, decisão, transferência e absorção de tecnologia, aquisição de produtos estratégicos para o SUS no âmbito das PDP e o respectivo monitoramento e avaliação. DOU. 13 nov. 2014 b, Sec. 1, p. 102.

MINISTÉRIO DA SAÚDE; RABELO, C. Avanços Estratégicos: Número de transplantes mais que dobra em dez anos. $<$ http://portalsaude.saude.gov.br/portalsaude/noticia/4234/162/numero-de-transplantes-mais-que-dobraem-dez-anos.html>. Acesso em: 7 out. 2013.

MS; SCTIE; DAF. Relação Nacional de Medicamentos Essenciais: RENAME 2014. 9. ed. Brasília: Ministério da Saúde, 2015.

MUELLER, L. L.; TAKETSUMA COSTA, S. M. Should ANVISA be permitted to reject pharmaceutical patent applications in Brazil? Expert Opinion on Therapeutic Patents, v. 24, n. 1, p. 1-4, jan. 2014.

MUSSKOPF, D. B. What Japan can teach us about PPH: Important features extracted from questionnarie survey. Tókio: Tokio Institute of Technology/ JPO, mar. 2012.

PLAZA, C. Á. Notas sobre patentes de novas formas polimórficas. Direito Contemporâneo e Constituição, v. 1, n. 1, p. 106-143, 30 dez. 2012.

PORTER, A. L.; CUNNINGHAM, S. W. Tech mining: exploiting new technologies for competitive advantage. 1. ed. EUA: Wiley. com, 2004.

SOARES, C. S.; CORONEL, D. A.; FILHO, P. J. M. A recente política industrial brasileira: da "política de desenvolvimento produtivo" ao "plano Brasil maior”. Revista Perspectivas Contemporâneas, v. 8, n. 1, p. 1-20, jun. 2013.

TOJAL, S. B. DE B.; PESSÔA, P. R. A anuência prévia na concessão de patentes de medicamentos e a regulação econômica da indústria farmacêutica. Revista de Direito Sanitário, v. 8, n. 3, p. 148-165, fev. 2008.

WALLS, H. L.; SMITH, R. D.; DRAHOS, P. Improving regulatory capacity to manage risks associated with trade agreements. Globalization and Health, v. 11, n. 1, dez. 2015.

WHO. WHO | Global Glossary on donation and transplantation. Institucional. Disponível em: <http://www.who.int/transplantation/activities/en/>. Acesso em: 18 fev. 2016.

As bases de informações Cortellis for Competitive Intelligence e Thomson Innovation são produtos de uso mediante assinatura junto a empresa Thomson Reuters.

\section{AGRADECIMENTOS}

As autoras agradecem ao Dr. Fernando Palop $↔$ - Universidad de Valencia, TRIZ XXI pelo treinamento e disponibilização do software Vantagepoint e a Anvisa pelo acesso as bases de informações de uso mediante assinatura. 